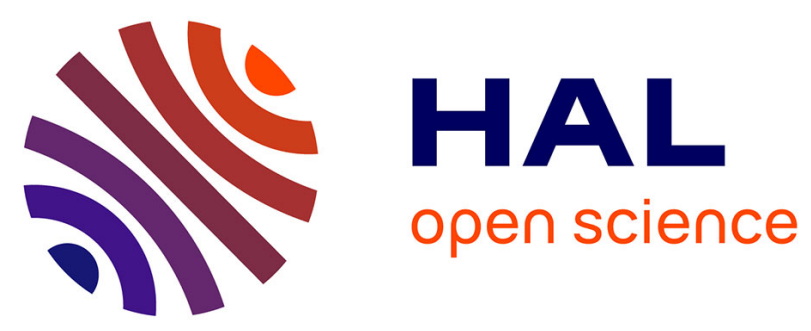

\title{
Optical sideband generation up to room temperature with mid-infrared quantum cascade lasers
}

S Houver, P Cavalié, M Renaudat St-Jean, M.I. Amanti, C Sirtori, L.H. Li, A.G. Davies, E.H. Linfield, T.A.S. Pereira, A Lebreton, et al.

\section{To cite this version:}

S Houver, P Cavalié, M Renaudat St-Jean, M.I. Amanti, C Sirtori, et al.. Optical sideband generation up to room temperature with mid-infrared quantum cascade lasers. Optics Express, 2015, 23 (4), pp.4012-4020. 10.1364/OE.23.004012 . hal-01275202

\section{HAL Id: hal-01275202 \\ https://hal.sorbonne-universite.fr/hal-01275202}

Submitted on 17 Feb 2016

HAL is a multi-disciplinary open access archive for the deposit and dissemination of scientific research documents, whether they are published or not. The documents may come from teaching and research institutions in France or abroad, or from public or private research centers.
L'archive ouverte pluridisciplinaire HAL, est destinée au dépôt et à la diffusion de documents scientifiques de niveau recherche, publiés ou non, émanant des établissements d'enseignement et de recherche français ou étrangers, des laboratoires publics ou privés.

\section{(c)(1)}

Distributed under a Creative Commons Attribution| 4.0 International License 


\title{
Optical sideband generation up to room temperature with mid-infrared quantum cascade lasers
}

\author{
S. Houver, ${ }^{1, *}$ P. Cavalié, ${ }^{1}$ M. Renaudat St-Jean, ${ }^{2}$ M. I. Amanti, ${ }^{2}$ C. Sirtori ${ }^{2}$ L. H. Li, ${ }^{3}$ A. \\ G. Davies, ${ }^{3}$ E. H. Linfield, ${ }^{3}$ T. A. S. Pereira, ${ }^{4}$ A. Lebreton, ${ }^{1}$ J. Tignon, ${ }^{1}$ S. S. Dhillon ${ }^{1}$ \\ ${ }^{1}$ Laboratoire Pierre Aigrain, Ecole Normale Supérieure-PSL Research University, CNRS, Université Pierre et Marie \\ Curie-Sorbonne Universités, Université Paris Diderot-Sorbonne Paris Cité, 24 rue Lhomond, 75231 Paris Cedex 05, \\ France \\ ${ }^{2}$ Laboratoire Matériaux et Phénomènes Quantiques, Université Paris Diderot, 75205 Paris Cedex 13, France \\ ${ }^{3}$ School of Electronic and Electrical Engineering University of Leeds, Woodhouse Lane, Leeds LS9 2JT, UK \\ ${ }^{4}$ Instituto de Física, Universidade Federal de Mato Grosso, 78060-900, Cuiabá Mato Grosso, Brazil \\ sarah.houver@lpa.ens.fr
}

\begin{abstract}
Mid-infrared (MIR) sideband generation on a near infrared (NIR) optical carrier is demonstrated within a quantum cascade laser (QCL). By employing an externally injected NIR beam, $E_{\mathrm{NIR}}$, that is resonant with the interband transitions of the quantum wells in the QCL, the nonlinear susceptibility is enhanced, leading to both frequency mixing and sideband generation. A GaAs-based MIR QCL $\left(E_{\mathrm{QCL}}=135 \mathrm{meV}\right)$ with an aluminumreinforced waveguide was utilized to overlap the NIR and MIR modes with the optical nonlinearity of the active region. The resulting difference sideband $\left(E_{\mathrm{NIR}}-E_{\mathrm{QCL}}\right)$ shows a resonant behavior as a function of NIR pump wavelength and a maximum second order nonlinear susceptibility, $\chi^{(2)}$, of $\sim 1 \mathrm{~nm} / \mathrm{V}$ was obtained. Further, the sideband intensity showed little dependence with the operating temperature of the QCL, allowing sideband generation to be realized at room temperature.
\end{abstract}

(C) 2015 Optical Society of America

OCIS codes: (140.0140) Lasers and laser optics; (140.5965) Semiconductor lasers, quantum cascade; (190.4223) Nonlinear wave mixing.

\section{References and links}

1. C. Phillips, M. Y. Su, M. S. Sherwin, J. Ko, and L. Coldren, "Generation of first-order terahertz optical sidebands in asymmetric coupled quantum wells," Appl. Phys. Lett. 75(18), 2728-2730 (1999).

2. W. Shin, S. Han, C. Park, and K. Oh, "All fiber optical inter-band router for broadband wavelength division multiplexing," Opt. Express 12(9), 1815-1822 (2004).

3. J. Kono, M. Y. Su, T. Inoshita, T. Noda, M. S. Sherwin, J. S. J. Allen, Jr., and H. Sakaki, "Resonant Terahertz Optical Sideband Generation from Confined Magnetoexcitons," Phys. Rev. Lett. 79(9), 1758-1761 (1997).

4. M. Wagner, H. Schneider, S. Winnerl, M. Helm, T. Roch, A. M. Andrews, S. Schartner, and G. Strasser, "Resonant enhancement of second order sideband generation for intraexcitonic transitions in GaAs/AlGaAs multiple quantum wells," Appl. Phys. Lett. 94(24), 241105 (2009).

5. S. G. Carter, V. Ciulin, M. S. Sherwin, M. Hanson, A. Huntington, L. A. Coldren, and A. C. Gossard, "Terahertz electro-optic wavelength conversion in GaAs quantum wells: Improved efficiency and room-temperature operation," Appl. Phys. Lett. 84(6), 840-842 (2004).

6. B. Zaks, R. B. Liu, and M. S. Sherwin, "Experimental observation of electron-hole recollisions," Nature 483(7391), 580-583 (2012).

7. R. Köhler, A. Tredicucci, F. Beltram, H. E. Beere, E. H. Linfield, A. G. Davies, D. A. Ritchie, R. C. Iotti, and F. Rossi, "Terahertz semiconductor-heterostructure laser," Nature 417(6885), 156-159 (2002).

8. A. Valavanis, J. Zhu, J. Freeman, L. Li, L. Chen, A. G. Davies, E. H. Linfield, and P. Dean, "Terahertz quantum cascade lasers with $>1$ W output powers," Electron. Lett. 50(4), 309-311 (2014).

9. P. Cavalié, J. Freeman, K. Maussang, E. Strupiechonski, G. Xu, R. Colombelli, L. Li, A. G. Davies, E. H. Linfield, J. Tignon, and S. S. Dhillon, "High order sideband generation in terahertz quantum cascade lasers," Appl. Phys. Lett. 102(22), 221101 (2013). 
10. J. Madéo, P. Cavalie, J. R. Freeman, N. Jukam, J. Maysonnave, K. Maussang, H. E. Beere, D. A. Ritchie, C. Sirtori, J. Tignon, and S. S. Dhillon, "All-optical wavelength shifting in a semiconductor laser using resonant nonlinearities," Nat. Photonics 6(8), 519-524 (2012).

11. J. Faist, F. Capasso, D. L. Sivco, C. Sirtori, A. L. Hutchinson, and A. Y. Cho, "Quantum Cascade Laser," Science 264(5158), 553-556 (1994).

12. F. Capasso, "High-performance midinfrared quantum cascade lasers," Opt. Eng. 49(11), 111102 (2010).

13. A. Lyakh, R. Maulini, A. Tsekoun, R. Go, C. Pflügl, L. Diehl, Q. J. Wang, F. Capasso, and C. K. N. Patel, “3 W continuous-wave room temperature single-facet emission from quantum cascade lasers based on nonresonant extraction design approach,” Appl. Phys. Lett. 95(14), 141113 (2009).

14. R. L. Sutherland, Handbook of Nonlinear Optics (Taylor \& Francis, 2003).

15. C. Zervos, M. D. Frogley, C. C. Phillips, D. O. Kundys, L. R. Wilson, J. W. Cockburn, M. Hopkinson, and M. S. Skolnick, "Coherent near-infrared wavelength conversion in semiconductor quantum cascade lasers," Appl. Phys. Lett. 89(18), 183507 (2006).

16. R. Boyd, Nonlinear Optics, 3rd Edition, (Elsevier, 2008).

17. G. Bastard, Wave mechanics applied to semiconductor heterostructures (Les Éditions de Physique, 1988).

18. H. Page, C. Becker, A. Robertson, G. Glastre, V. Ortiz, and C. Sirtori, "300 K operation of a GaAs-based quantum-cascade laser at $\lambda \approx 9 \mu \mathrm{m}$," Appl. Phys. Lett. 78(22), 3529-3531 (2001).

19. E. Rosencher, A. Fiore, B. Vinter, V. Berger, P. Bois, and J. Nagle, "Quantum Engineering of Optical Nonlinearities," Science 271(5246), 168-173 (1996).

20. J. Khurgin, "Second-order nonlinear effects in asymmetric quantum-well structures," Phys. Rev. B Condens. Matter 38(6), 4056-4066 (1988).

21. A. K. Saxena, "Electron mobility in Ga(1-x)Al(x)As alloys," Phys. Rev. B 24(6), 3295-3302 (1981).

22. J. I. Pankove, “Absorption Edge of Impure Gallium Arsenide,” Phys. Rev. 140(6A), A2059-A2065 (1965).

23. S. S. Dhillon, C. Sirtori, J. Alton, S. Barbieri, A. de Rossi, H. E. Beere, and D. A. Ritchie, "Terahertz transfer onto a telecom optical carrier," Nat. Photonics 1(7), 411-415 (2007).

24. M. A. Afromowitz, "Refractive index of Ga1-xAlxAs," Solid State Commun. 15(1), 59-63 (1974).

25. J. V. D. Veliadis, J. B. Khurgin, Y. J. Ding, A. G. Cui, and D. S. Katzer, "Investigation of the photoluminescence-linewidth broadening in periodic multiple narrow asymmetric coupled quantum wells," Phys. Rev. B Condens. Matter 50(7), 4463-4469 (1994).

26. H. Yoshida, T. Mozume, T. Nishimura, and O. Wada, "Ultrafast intersubband transitions in InGaAs/AlAs coupled double quantum well structures for near-infrared all-optical switching," in Lasers and Electro-Optics Society Annual Meeting, 1997. LEOS '97 10th Annual Meeting. Conference Proceedings., IEEE, 1997), 441-442 vol.442.

27. C. Y. Wang, L. Kuznetsova, V. M. Gkortsas, L. Diehl, F. X. Kärtner, M. A. Belkin, A. Belyanin, X. Li, D. Ham, H. Schneider, P. Grant, C. Y. Song, S. Haffouz, Z. R. Wasilewski, H. C. Liu, and F. Capasso, "Mode-locked pulses from mid-infrared quantum cascade lasers," Opt. Express 17(15), 12929-12943 (2009).

28. A. Hugi, G. Villares, S. Blaser, H. C. Liu, and J. Faist, "Mid-infrared frequency comb based on a quantum cascade laser," Nature 492(7428), 229-233 (2012).

29. M. G. Hansen, I. Ernsting, S. V. Vasilyev, A. Grisard, E. Lallier, B. Gérard, and S. Schiller, "Robust, frequencystable and accurate mid-IR laser spectrometer based on frequency comb metrology of quantum cascade lasers up-converted in orientation-patterned GaAs," Opt. Express 21(22), 27043-27056 (2013).

\section{Introduction}

Coherent terahertz-optical sideband generation based on resonant nonlinear effects has been previously investigated [1] for potential applications in frequency modulation (FM) and amplitude modulation (AM) of an optical carrier wave at terahertz (THz) rates, as well as for provision of large all-optical wavelength shifts between telecommunication bands [2]. Sideband generation typically involves a near-infrared $\left(E_{\mathrm{NIR}}\right)$ excitation that is mixed with a $\mathrm{THz}$ beam $\left(E_{\mathrm{THz}}\right)$ within an optical nonlinear material i.e. $E_{\text {sideband }}=E_{\mathrm{NIR}} \pm \mathrm{n} E_{\mathrm{THz}}$ where $\mathrm{n}$ is an integer $(\mathrm{n}=1,2,3$ etc.). A strong $\mathrm{THz}$ source, however, is required for the observations of sidebands. Indeed, sideband generation has been observed in III-V based semiconductors using a free electron laser (FEL) combined with resonant nonlinearities [3, 4] with high conversion efficiencies and room temperature operation [5] as well as extension to high-order harmonic generation [6].

THz quantum cascade lasers (QCLs) [7] are compact and practical semiconductor devices based on intersubband transitions within quantum wells and over the last decade have been developed to give high output powers [8] with intra-cavity powers approaching those used in FEL-based studies. We have recently demonstrated $[9,10]$ that sideband generation (including high orders) can be realized using the combined resonant interband and intersubband nonlinearities of $\mathrm{THz}$ QCLs. However, these investigations have been 
performed at low temperatures $(\sim 10 \mathrm{~K})$ that have been necessary to achieve the highest intracavity powers. Furthermore, the wavelength shifts realized were small, limited by the QCL photon energy, which restricts the application of QCLs for wavelength shifting.

In this paper we demonstrate nonlinear sideband generation using a mid-infrared (MIR) QCL [11] and a resonant NIR field. This enables operation at room temperature, as well as providing large wavelength shifts. MIR QCLs are considerably more powerful than their $\mathrm{THz}$ frequency counterparts [12] and operate at higher temperatures [13]. However, owing to a much smaller refractive index compared to $\mathrm{THz}$ frequencies, MIR QCLs have a considerably greater phase-mismatch with the NIR that is comparable in absolute values to the interband losses, with both contributing to a reduction in the sideband efficiency [14]Here we show that, using resonant nonlinearities in a three level system, we can compensate for this phasemismatch. This is realized through a new waveguide that is reinforced with high contrast dielectric layers that ensures that the interband excitation and the sideband are guided within the active region, leading to efficient excitation of the resonant second order nonlinearity, $\chi^{(2)}$.

Previously, MIR optical sideband generation has been demonstrated in QCLs by Zervos et al [15]. However, the observed sideband was extremely weak and only seen at $15 \mathrm{~K}$ with a limited resolution owing to the geometry of the apparatus that resulted in strong absorption of the generated sideband. In our work high resolution MIR sideband generation is observed up to room temperature through a slightly detuned resonant interaction allowing a compromise between the enhanced nonlinearity and the increased optical losses to be obtained. We also show that the resonant nonlinear susceptibility is one order of magnitude larger than that of bulk (i.e. non-resonant) GaAs.

\section{Resonant Process and Geometry}

A resonant nonlinearity corresponds to an enhancement of $\chi^{(2)}$ when one or more incident electromagnetic fields are at exactly the same energy as a material transition. In the case of difference frequency generation in a three level system (Fig. 1(a)), two contributions are possible: a single or a double resonance [16]. For the former, only one of the excitations, $E_{\mathrm{a}}$, is resonant with a real transition, $\Delta E_{12}$ :

$$
\chi_{\text {single }}^{(2)} \propto \frac{1}{E_{a}-\Delta E_{12}-i \Gamma_{12}} \propto \frac{1}{\Gamma_{12}}
$$

where $\Gamma_{12}$ is the linewidth of the transition. For the latter, all the excitations, $E_{\mathrm{a}}, E_{\mathrm{b}}$ and $E_{\mathrm{c}}$ are resonant with the transitions $\Delta E_{13}, \Delta E_{12}$ and $\Delta E_{23}$, and $\chi^{(2)}$ is given by:

$$
\chi_{\text {double }}^{(2)} \propto \frac{1}{\left(E_{a}-\Delta E_{13}-i \Gamma_{13}\right)} \frac{1}{\left(E_{b}-\Delta E_{12}-i \Gamma_{12}\right)} \propto \frac{1}{\Gamma_{13} \Gamma_{12}}
$$

This results in a much larger nonlinearity when compared to a single resonance. A MIR QCL, however, has a more complex bandstructure than a simple three level system. Figure 1(b) shows the schematic of the resonant nonlinear process for the generation of the difference frequency, similar to that observed in sideband generation with THz QCLs [10]. One hole state and the three principal electronic states in the active region of the QCL are shown: the MIR QCL transition, $E_{\mathrm{QCL}}$ (represented in green), takes place between the $E_{3}$ and $E_{2}$ states (represented in green) with the lower state $E_{2}$ depopulated by resonant electron-phonon scattering to the electron state $E_{1}\left(E_{\mathrm{LO}}=E_{2}-E_{1} \sim 36 \mathrm{meV}\right)$. The external NIR excitation, $E_{\mathrm{NIR}}$ (blue arrow) is brought into resonance with interband transitions between the confined hole and the lowest lying electronic states $\left(E_{2}\right.$ and $\left.E_{1}\right)$. This resonance enhances the nonlinear susceptibility, permitting a nonlinear interaction between the NIR and MIR beams within the QCL cavity and resulting in sideband generation through the difference frequency, $E_{\text {sideband }}=$ $E_{\mathrm{NIR}}-E_{\mathrm{QCL}}$ (red arrow). 


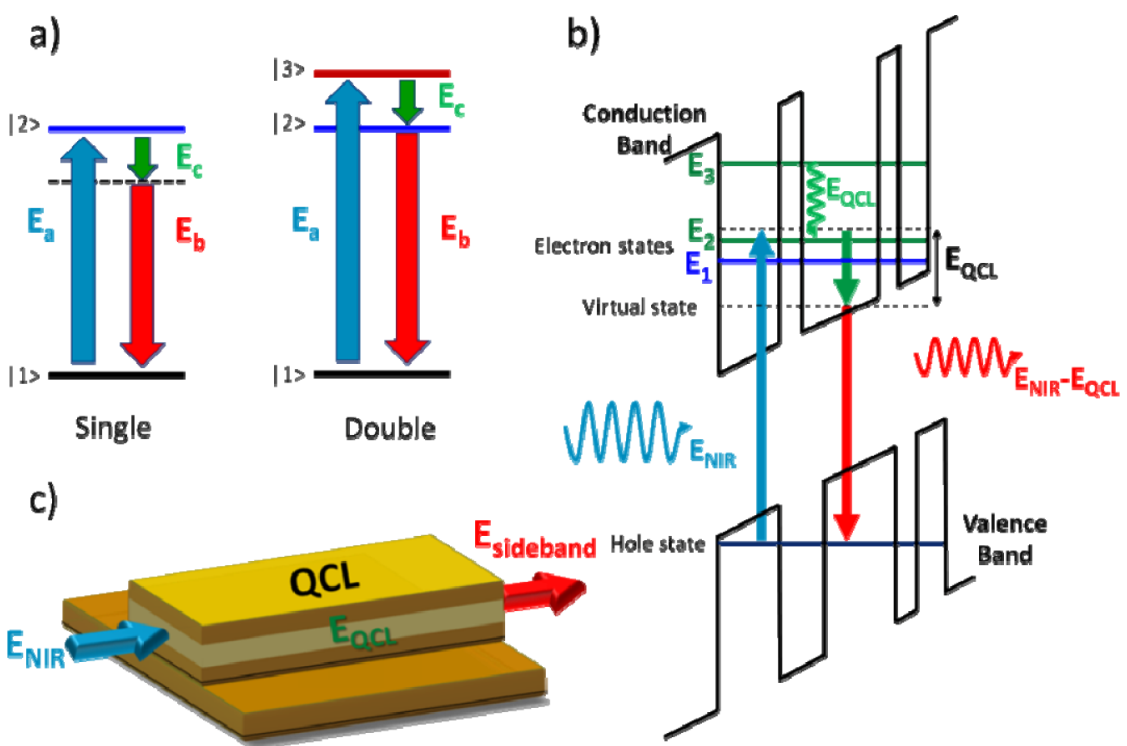

Fig. 1. (a) Schematic diagrams showing a single and a double resonant nonlinear process (b) Schematic diagram of a MIR QCL showing the resonant nonlinear process for sideband generation $\left(E_{\mathrm{NIR}}-E_{\mathrm{QCL}}\right)$, represented in three quantum wells for clarity. The QCL is operating at $E_{\mathrm{QCL}}$ (green wavy arrow between upper state $E_{3}$ and lower state $E_{2}$ ). The lower state $E_{2}$ is depopulated by LO-phonons to electron state $E_{1}$. A NIR pump ( $E_{\mathrm{NIR}}$, blue arrow) is tuned in resonance with the interband transitions. This results in the generation of a lower-energy beam (red arrow) at $E_{\mathrm{NIR}}-E_{\mathrm{QCL}}$. (c) Schematic of collinear geometry with the input excitation coupled into one facet of the QCL and the sideband exiting the opposite facet.

Our experimental realization of the sideband generation scheme is based on a collinear geometry for all the interacting waves [10] i.e. in the same plane parallel to the surface of the QCL (Fig. 1(c)). This type of guided geometry for both the MIR emission and input interband excitation provides a relatively long interaction length $\left(\sim 1 / \alpha_{\mathrm{p}}\right.$ where $\alpha_{\mathrm{p}}$ are the pump losses) compared to surface excitation [15]. The QCL also acts as an optical filter of the NIR pump when in resonance with interband transitions, facilitating the observation of the sideband. Experimentally, the NIR pump is coupled into one end of the QCL cavity (the facet) and the sideband exits the opposite facet (Fig. 1(c)), with the NIR pump absorbed if above the effective bandgap of the material (i.e. the lowest lying electron-hole transitions). Similarly for this transmission geometry, the generated sideband can only be observed if it lies below the effective band gap as otherwise it would be absorbed. Hence only the lowest energy interband resonances can be explored. In this case the full expression for the resonant $\chi^{(2)}$ for a three level nonlinear interaction becomes $[16,17]$ :

$$
\chi^{(2)}=\frac{N_{H}}{\varepsilon_{0}} \frac{\mu_{H E_{1}} \mu_{E_{1} E_{2}} \mu_{E_{2} H}}{\left(E_{\text {sideband }}-\Delta E_{H E_{1}}-i \Gamma_{H E_{1}}\right)\left(E_{N I R}-\Delta E_{H E_{2}}-i \Gamma_{H E_{2}}\right)}
$$

where, $E_{\mathrm{NIR}}$ is detuned from $\Delta E_{\mathrm{HE} 2}$ (transition between the hole state and $\mathrm{E}_{2}$ ) by $E_{\mathrm{QCL}}-E_{\mathrm{LO}}$ $\sim 100 \mathrm{meV}$ resulting in a reduction in $\chi^{(2)}$. $E_{\text {sideband }}$ is close to resonance with $\Delta E_{\mathrm{HE} 1}$, the transition between the hole state and $E_{1} \cdot \mu_{\mathrm{ij}}\left(\mathrm{E}_{\mathrm{ij}}\right)$ are the dipole matrix elements (transitions energies) for the 3 states $\left(H, E_{1}\right.$ and $\left.E_{2}\right)$ and $N_{\mathrm{H}}$ is the density of states is the valence band.

\section{QCL design}

GaAs/AlGaAs based QCLs grown by molecular beam epitaxy (MBE) employing a three-well active region (AR) design [18] were utilized. This design has been previously used to demonstrate high temperature laser operation at $\sim 9.2 \mu \mathrm{m}\left(v_{\mathrm{QCL}}=33 \mathrm{THz}, E_{\mathrm{QCL}}=135 \mathrm{meV}\right)$. 
The AR comprises a total of 45 periods resulting in an AR thickness of $2 \mu \mathrm{m}$, with each period n-doped to $1.4 \times 10^{17} \mathrm{~cm}^{-3}$. In order to achieve resonant frequency mixing it is important to consider both the waveguide modes of the MIR QCL emission and the external NIR excitation. Figure 2(a) shows these optical guided modes in the typical plasmonenhanced waveguides used in GaAs-based MIR QCLs [18]. Here both sides of the AR are clad by thick low doped GaAs layers (C2), which are themselves clad by highly-doped GaAs (the 'plasmon' layers, $\mathrm{C} 1$ ) to reduce the MIR refractive index and hence confine the MIR mode. The MIR, the NIR modes and the NIR refractive index are shown in green, red and blue, respectively. The MIR mode is well confined in the AR as expected. However, the two fundamental NIR modes are confined on either side of the AR owing to the larger refractive index of GaAs compared with that of the AR. Even though there is an overlap of the modes, a spatial overlap between the modes and the AR is necessary to obtain frequency mixing. This is a result of inversion symmetry arguments - GaAs does not possess a large second order resonant nonlinearity unless there is an asymmetry in the electronic potential, for example when a large electric field is applied to quantum wells [19], or in asymmetric coupled quantum wells [20], as in the AR. Indeed sideband generation was not detected with a QCL with this type of waveguide.

a)

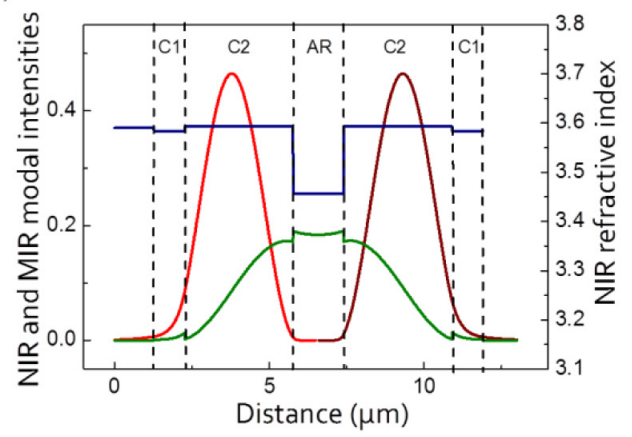

b)

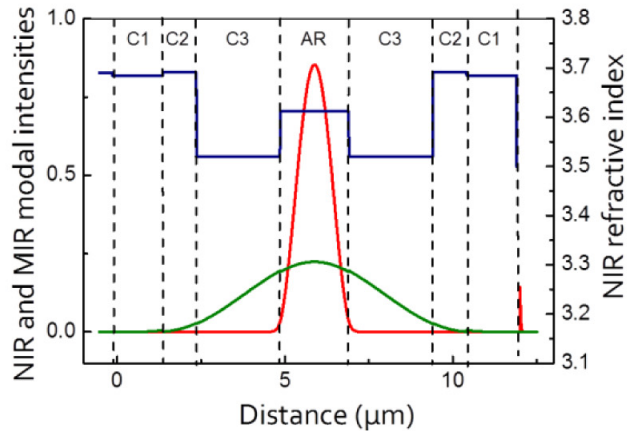

Fig. 2. Intensity profiles of the MIR QCL mode at $9.2 \mu \mathrm{m}$ (green line) and the NIR modes at $800 \mathrm{~nm}$ (red lines). The right axes show the NIR refractive index of the layers (dark blue line). (a) Conventional plasmon enhanced MIR waveguide with GaAs cladding layers ( $\mathrm{C} 1$ doped at 6 $\times 10^{18} \mathrm{~cm}^{-3}$ and $\mathrm{C} 2$ doped at $8 \times 10^{16} \mathrm{~cm}^{-3}$ ) around the AR. (b) Modified Al-enhanced MIR waveguide with the additional $\mathrm{Al}_{0.25} \mathrm{Ga}_{0.75} \mathrm{As}$ layers (C3) around the AR to confine the NIR mode in the AR. C3 is doped at $1 \times 10^{17} \mathrm{~cm}^{-3}$. $\mathrm{C} 1$ is identical to (a) whilst $\mathrm{C} 2$ is GaAs doped at $1 \times 10^{18} \mathrm{~cm}^{-3}$.

To this end, a new waveguide was designed that has been reinforced by the insertion of $\mathrm{Al}_{0.25} \mathrm{Ga}_{0.75}$ As layers directly around the AR (C3 in Fig. 2(b)). As shown on the right axis of Fig. 2(b), the refractive index of this layer is lower than that of the AR which has an average aluminium-content of $\sim 17 \%$. This allows the NIR mode to be guided within the active region with a $100 \%$ overlap, and an overlap of $43 \%$ with the MIR mode ensuring that a resonant nonlinearity is induced for sideband generation. The MIR QCL mode itself is slightly improved upon incorporation of the $\mathrm{Al}_{0.25} \mathrm{Ga}_{0.75} \mathrm{As}$ layers with an $\mathrm{AR}$ overlap and losses of $43 \%$ and $15 \mathrm{~cm}^{-1}$ compared to $34 \%$ and $15.6 \mathrm{~cm}^{-1}$, respectively, for a conventional waveguide. In principal, an increase in confinement would be obtained by increasing the aluminium content further but $25 \%$ was chosen as a compromise between the increase in the refractive index contrast and the reduction in the electronic mobility [21].

The samples were processed, cleaved and mounted in a continuous flow cryostat on copper mounts, where the temperature was varied between $210 \mathrm{~K}$ and room temperature. At $210 \mathrm{~K}$ the samples demonstrated a laser threshold current density of $10 \mathrm{kA} / \mathrm{cm}^{2}$ with peak output powers of $95 \mathrm{~mW}$ (inset to Fig. 5). 
The external NIR excitation was provided by continuous wave tunable Ti:Sapphire (Ti:Sa) laser $(\lambda=750 \mathrm{~nm}-900 \mathrm{~nm})$ with output powers of $10 \mathrm{~mW}$. (These low pumps powers result in a small excited population near the input facet which does not alter the threshold or output powers of the QCL). The polarization was chosen to be the same as for the QCL emission i.e. transverse magnetic (TM) and thus will predominately excite transitions from light hole states [10]. The NIR beam was coupled into and out of the QCL cavity using high numerical aperture objectives. The transmitted difference frequency was then detected using a grating spectrometer coupled to a CCD array. The QCL was operated in pulsed mode with $150 \mathrm{~ns}$ pulses and at a frequency of $70 \mathrm{kHz}$, and the Ti:Sa laser was synchronized to the QCL modulation using an acousto-optic modulator.

\section{MIR sideband generation}

Figure 3 shows the transmitted signal detected on the CCD array with the QCL biased above the laser threshold at a temperature of $210 \mathrm{~K}$. The QCL ridge width was $12 \mu \mathrm{m}$ with a cavity length of $1 \mathrm{~mm}$. The NIR excitation, $E_{\mathrm{NIR}}$, was $1.61 \mathrm{eV}$, considerably above the lowest electron-hole transition of the material (the effective bandgap, $\left.\mathrm{E}_{\mathrm{g}} \sim 1.52 \mathrm{eV}\right)$. ( $E_{\mathrm{NIR}}$ is visible as a result of a parasitic part of the beam that does not pass through the ridge). The sideband is clearly observed at $E_{\text {sideband }}=E_{\mathrm{NIR}}-E_{\mathrm{QCL}}=1.475 \mathrm{eV}\left(\lambda_{\text {sideband }}=840 \mathrm{~nm}\right)$, i.e. separated from the pump $E_{\mathrm{NIR}}$ by the QCL photon energy $\left(E_{\mathrm{QCL}}=135 \mathrm{meV}\right)$ and is below $E_{\mathrm{g}}$. The inset shows an amplified view of the sideband revealing many modes with a $0.2 \mathrm{meV}$ separation ( 48 $\mathrm{GHz}$ ), and is a replica of the QCL Fabry Perot emission.

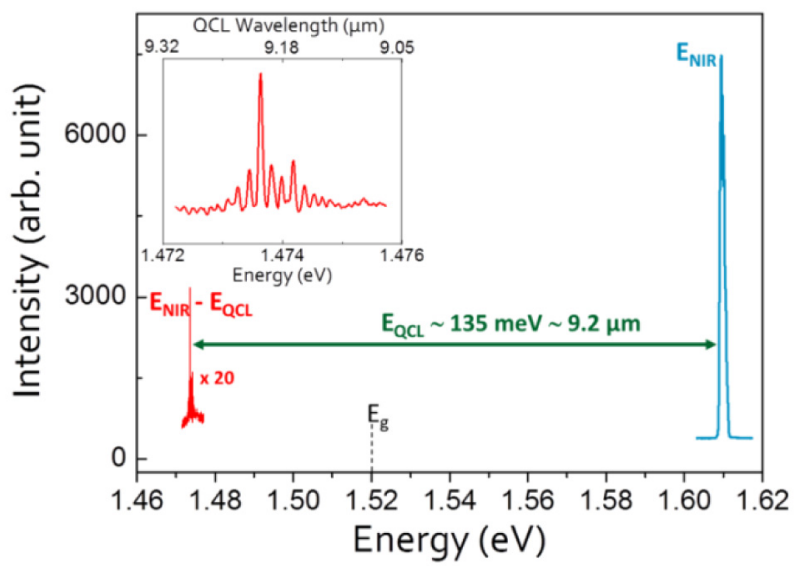

Fig. 3. Sideband spectrum measured with the QCL at a temperature of $210 \mathrm{~K}$. The NIR excitation beam is at $1.61 \mathrm{eV}$, above the effective bandgap, $E_{\mathrm{g}}$, and the generated sideband is observed at $1.475 \mathrm{eV}$, separated from the NIR beam by exactly the MIR QCL energy (135 $\mathrm{meV})$. The inset shows an expanded view of the generated sideband. The top axis of the inset is the calculated MIR spectral range ( $\left.E_{\mathrm{NIR}}-E_{\text {sideband }}\right)$.

To demonstrate the resonant nature of the sideband generation, the integrated sideband intensity was investigated as a function of the pump excitation energy (Fig. 4(a) - black squares). As the NIR beam is increased in energy above $E_{g}$, the sideband intensity slowly increases. A clear maximum is observed for a pump energy of $1.61 \mathrm{eV}\left(E_{\text {sideband }}=1.475 \mathrm{meV}\right)$ followed by sharp drop in intensity. The latter is a result of the sideband being absorbed as its energy starts to exceed the effective bandgap of the material for pump energies greater than $1.61 \mathrm{eV}$ owing to an exponential increase in the losses. The data greater than $1.61 \mathrm{eV}$ is fitted with an exponential fit to illustrate the sharp drop in sideband intensity [22]. The lower energy part of the curve can be fitted by a lorentzian dependence centered on the peak pump energy. This highlights the dependence of the sideband intensity on the square of the resonant 
susceptibility and how it increases rapidly as a resonance is approached. In the case of a single resonance [16]:

$$
I_{\text {sideband }} \propto\left|\chi^{(2)}\right|^{2} \propto \frac{1}{\left(E_{N I R}-E_{0}\right)^{2}-\Gamma^{2}}
$$

where $E_{0}$ is the energy of the peak intensity $(1.613 \mathrm{eV}$ from the fit of the data) and $2 \Gamma$ is the full-width-at-half maximum of the nonlinearity (equal to $82 \mathrm{meV}$ from the fit of the data). Although a lorentzian fit can be performed, the number of states involved is greater than just a single resonance.

Figure 4(b) shows the bandstructure of the QCL transition zone (where the electronic transition takes place) with both the valence and conduction band profiles. The main states are highlighted: the two lowest hole states $L H_{1}$ and $L H_{2}$ and the electron states $E_{3}, E_{2}$ and $E_{1}$. The laser transition is $E_{3}-E_{2} \sim 135 \mathrm{meV}$ while $E_{2}-E_{1} \sim 36 \mathrm{meV}$ is equal to the GaAs phonon energy. As the interband dipole matrix elements [Eq. (3)] are proportional to the overlap integrals between the hole and electron states, $\left|\left\langle\Psi_{\mathrm{H}} \mid \Psi_{\mathrm{E}}\right\rangle\right|[16]$, the latter are compared to the sideband intensity in Fig. 4(a) (red stars). In the energy range before the onset of absorption of the sideband, significant overlaps are observed for the transitions $L H_{1} E_{1}, L H_{1} E_{2}$ and $L H_{2} E_{1}$ at $1.549 \mathrm{eV}, 1.586 \mathrm{eV}$ and $1.605 \mathrm{eV}$ respectively. It appears that $L H_{1} E_{2}$ and $L H_{2} E_{1}$ coincide with the increase in sideband intensity until absorption of the sideband sets in, with little or no contribution to the sideband from the $L H_{1} E_{1}$ excitation. However, as the interband excitation is above the effective gap, a simple two level consideration is insufficient to describe the system and a three level system needs to be considered instead. Indeed, using [Eq. (3)] summed over the contribution of both hole states, $\left|\chi^{(2)}\right|^{2}$ is calculated and plotted in Fig. 4(a), and shows a good correlation with the experimental data until the absorption point of the generated sideband. ( $\boldsymbol{\Gamma}$ was chosen to be $40 \mathrm{meV}$ from the lorentzian fit and $N_{\mathrm{H}}$ (the density of light hole states is the valence band) $\sim 3 \times 10^{17} \mathrm{~cm}^{-3}$ at $210 \mathrm{~K}$ [17]). The nonlinear susceptibility here is determined to be $\chi^{(2)}=0.8 \times 10^{3} \mathrm{pm} / \mathrm{V}$ and is compared to the experimentally determined value below.

a)

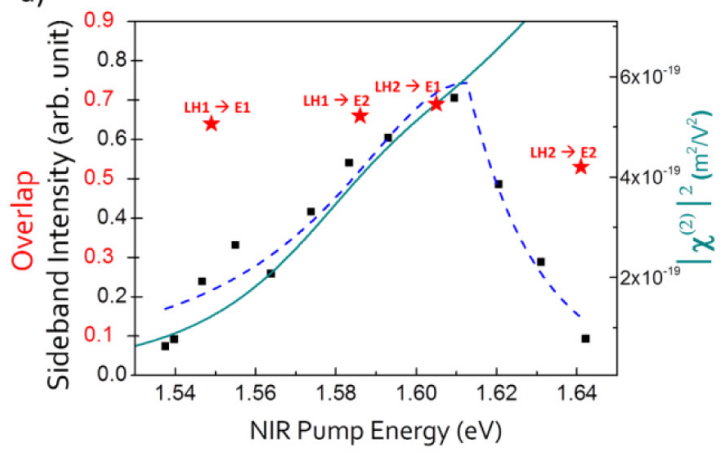

b)

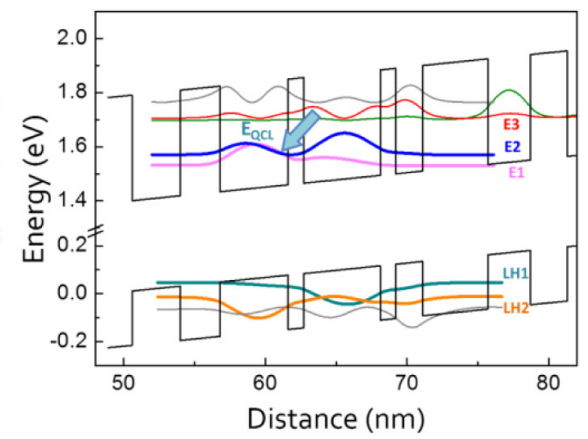

Fig. 4. (a) Integrated sideband intensity as a function of NIR pump energy (black dots). The dashed curve corresponds to a lorentzian fit to the lower energy part $\left(E_{\mathrm{NIR}}<1.61 \mathrm{eV}\right)$ of the data and an exponential fit for high energies $\left(E_{\mathrm{NIR}}>1.61 \mathrm{eV}\right)$. The electron-hole overlap integrals of the various transitions within the QCL are plotted as red stars. The right axis (green curve) shows the calculated $\left|\chi^{(2)}\right|^{2}$ from [Eq. (3)]. (b) QCL band structure, showing the valence and conduction bands and the squares of the main wavefunctions. The principal states involved in the nonlinear interaction are the electron states $E_{1}$ and $E_{2}$ and light hole states $L H_{1}$ and $L H_{2}$. $E_{3}$ (red) is the upper state of the QCL transition and $E_{2}$ (blue) is the lower state from which electrons relax to state $E_{1}$ (pink) by a LO phonon transition.

The efficiency of the sideband generation can be evaluated by measuring the integrated power at the sideband frequency and comparing it to the transmitted pump power when the 
pump energy is below the bandgap (i.e. $P_{\text {sideband }} / P_{\mathrm{NIR}}$ ) [10]. (This method allows the coupling efficiencies of the pump laser to be taken into account). A maximum efficiency of $1 \times 10^{-3 \%}$ was determined at $210 \mathrm{~K}$ for $E_{\mathrm{NIR}}=1.61 \mathrm{eV}$. This value is considerably lower than the $0.1 \%$ efficiencies obtained with THz QCLs [10] and can be attributed to a lower susceptibility and a larger phase mismatch for interactions with MIR radiation. Although the phase mismatch in THz QCLs could be neglected when compared to the optical interband losses $\left(\Delta k<<\alpha_{\mathrm{p}}\right)$ as a result of the large refractive index in the THz range [10,23], this is not the case in the MIR where the much lower refractive index results in a larger phase mismatch $\left(\Delta k \approx \alpha_{\mathrm{p}}\right)$. This limits further the interaction length, and as a result reduces the efficiency. Using the full expression for the nonlinear efficiency, $\eta$, the resonant nonlinear susceptibility can be determined from [14]:

$$
\eta=\frac{P_{\text {sideband }}}{P_{\text {NIR }}}=\frac{8 \pi^{2} P_{Q C L} L^{2}}{\varepsilon_{0} n_{\text {NIR }} n_{Q C L} n_{\text {sideband }} c S \lambda_{\text {sideband }}^{2}}\left|\chi^{(2)}\right|^{2} \frac{\sin ^{2}\left(\Delta k \frac{L}{2}\right)+\operatorname{sh}^{2}\left(\alpha_{p} \frac{L}{4}\right)}{\left(\Delta k \frac{L}{2}\right)^{2}+\left(\alpha_{p} \frac{L}{4}\right)^{2}} e^{-\alpha_{p} \frac{L}{2}}
$$

where $\mathrm{P}_{\text {sideband }}\left(\mathrm{n}_{\text {sideband }}\right), \mathrm{P}_{\mathrm{NIR}}\left(\mathrm{n}_{\mathrm{NIR}}\right), \mathrm{P}_{\mathrm{QCL}}\left(\mathrm{n}_{\mathrm{QCL}}\right)$ are the intensities (refractive indexes) of the difference frequency generated beam, the NIR input pump and the MIR QCL, respectively. $\lambda_{\text {sideband }}$ is the generated wavelength $(840 \mathrm{~nm}), \mathrm{S}$ is the interaction area determined from the modal overlap $\left(32 \mu \mathrm{m}^{2}\right), \Delta k$ is the phase mismatch and $\alpha_{\mathrm{p}}$ are the pump losses. Using the GaAs refractive index [24], $\Delta k$ is approximately $7000 \mathrm{~cm}^{-1}$ which is the same order of magnitude as $\alpha_{\mathrm{p}} \sim 3000 \mathrm{~cm}^{-1}$. Taking the maximum efficiency of $1 \times 10^{-3 \%}$, a second-order susceptibility of $\chi^{(2)} \sim 1 \times 10^{3} \mathrm{pm} / \mathrm{V}$ is determined. This is in reasonable agreement with the theoretical value above, considering that higher conduction band states are not taken into account and there is uncertainty in the phase mismatch. As with the efficiency, the susceptibility is smaller than in the THz range [10], but can be understood by the detuning of the MIR interaction from a fully resonant excitation, where $\chi^{(2)}$ is given by [Eq. (3)]. In the case of sideband generation with a THz QCL [10], a bound-to-continuum design was employed. That design has an electronic transition close to the absorption edge and can therefore be approximated by a simple three level system with a double resonant excitation with $E_{\mathrm{HE} 3} \sim E_{\mathrm{NIR}}, E_{\mathrm{HE} 2} \sim E_{\text {sideband }}$ and $E_{\mathrm{E} 2 \mathrm{E} 3}=E_{\mathrm{QCL}}$. However, for the case of the MIR QCL used here, the QCL transition is separated from the band-edge by the phonon energy between states $E_{1}$ and $E_{2}(36 \mathrm{meV})$. This results in a more complex system with a detuning from a fully resonant system, resulting in a lower susceptibility. It should be noted that if a double resonance can be achieved when all the beams are at resonance with real states, an order of magnitude increase in the susceptibility could be achieved, which would result in a much larger sideband efficiency. In this case the $k$-space dispersion [19] and the inclusion of the QCL state populations would need to be taken into account.

\section{Room temperature sidebands}

The temperature evolution of the sideband signal is shown in Fig. 5, for a $1.5 \mathrm{~mm}$ long QCL with a $7 \mu \mathrm{m}$ ridge together with the light-current (LI) characteristics of the lasers (inset). The output power was measured using a calibrated detector. The sideband intensity is plotted as a function of temperature (black squares), and compared to the maximum QCL output power (red squares). (The NIR pump energy was reduced for each temperature to take into account the reduction in the effective bandgap as the temperature is increased). Laser action is observed up to $300 \mathrm{~K}$ and sideband generation was observed up to $295 \mathrm{~K}$. The temperature dependence appears to be the same for both the sideband intensity and output power, suggesting that the resonant nonlinearity remains identical and that broadening of the related transitions is not critical between $210 \mathrm{~K}$ and $295 \mathrm{~K}$. This is possibly a result of the compensation of the broadening with an increase in the density of states with temperature 
[25]. These results are extremely promising for the future use of InGaAs/AlInAs//InP based QCLs that are considerably more powerful and exhibit higher temperature operation that their GaAs counterparts [13]. As the bandgap of InGaAs falls into the telecom range, this could lead to efficient sideband generation with MIR QCLs for communications applications.

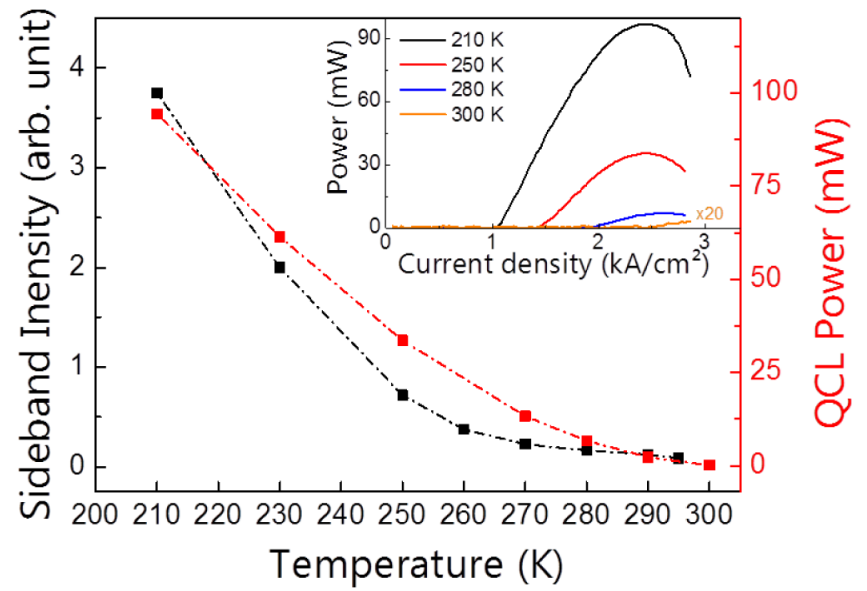

Fig. 5. Integrated spectra of each sideband as a function of temperature up to $295 \mathrm{~K}$ (black squares) compared with the QCL output power (red squares). Inset: Light-current characteristics for different temperatures.

\section{Conclusion}

Optical sideband generation has been demonstrated in MIR QCLs up to room temperature. By bringing a NIR interband excitation into resonance with the electronic states of a QCL, the nonlinear susceptibility is enhanced, resulting in frequency mixing and the generation of the difference frequency sideband. This was achieved by realizing an Al-reinforced MIR QCL waveguide to enable a strong overlap of all the interacting fields with the nonlinear active region. An efficiency of $1 \times 10^{-3 \%}$ was determined corresponding to a resonant second order nonlinearity of $1 \times 10^{3} \mathrm{pm} / \mathrm{V}$, which is one order of magnitude larger than the non-resonant bulk nonlinearity. Future work will concentrate on InP-based QCLs allowing sideband generation to be demonstrated in the telecommunications range, as well as increasing the conversion efficiency using a double resonant geometry. In addition to providing large wavelength shifts in the telecom bands between $1.3 \mu \mathrm{m}$ and $1.55 \mu \mathrm{m}$, the ultrafast properties of intersubband transitions [26] can be used to for sideband modulation at high frequencies (> $30 \mathrm{GHz}$ ) by direct electrical modulation of the QCL. Furthermore this technique will enable use of mature and high performance NIR techniques (such as fast and sensitive detectors) to be applied to the characterization of QCL for modelocking [27] or frequency comb generation [28], as well as providing a simple method of stabilizing a QCL to an optical comb through MIR up-conversion [29].

\section{Acknowledgements}

We acknowledge financial support from the French National Research Agency (ANR-13BS03-0001 "RE-LINQ"). The LPA-ENS is a "Unité Mixte de Recherche Associée au CNRS UMR8551 et aux Universités Paris 6 et 7". We are grateful for support from the EPSRC (UK), the Royal Society and Wolfson Foundation, and the ERC programme TOSCA. T. A. S. Pereira was financially supported by CAPES under process 3299/13-9. 